\title{
EFEITO AGUDO DO EXERCÍCIO AERÓBIO NA GLICEMIA EM DIABÉTICOS 2 SOB MEDICAÇÃO
}

\author{
ACUTE EFFECT OF AEROBIC EXERCISE ON BLOOD GLUCOSE IN DIABETIC 2 UNDER MEDICATION
}

EFECTO AGUDO DEL EJERCICIO AERÓBICO SOBRE LA GLUCEMIA EN LOS DIABÉTICOS 2

Artigo Original

Original Article ENMEDICACIÓN

\author{
Silvia Regina Barrile \\ (Fisioterapeuta) \\ Camila Borin Coneglian' \\ (Fisioterapeuta) \\ Camila Gimenes' \\ (Fisioterapeuta) \\ Marta Helena Souza de Conti ${ }^{1}$ \\ (Fisioterapeuta) \\ Eduardo Aguilar Arca' \\ (Fisioterapeuta) \\ Geraldo Rosa Junior' \\ (Fisioterapeuta) \\ Bruno Martinelli ${ }^{i}$ \\ (Fisioterapeuta) \\ 1 Universidade Sagrado Coração, \\ Centro de Ciências da Saúde, Curso \\ de Fisioterapia, Bauru, SP, Brasil.
}

\section{Correspondência:}

Rua Irmã Arminda, 10-50, Jardim Brasil, 17011-160, Bauru, SP, Brasil. srbarrile@gmail.com

\section{RESUMO}

Introdução: O exercício físico tem sido proposto como tratamento não farmacológico do diabetes por seu efeito hipoglicemiante. Objetivo: Verificar o efeito agudo do exercício sobre a glicemia capilar em indivíduos diabéticos que fazem uso de insulina ou antidiabéticos orais. Métodos: Foram estudados diabéticos em uso de hipoglicemiantes orais ( $G 1, n=7)$, não diabéticos ( $G 2, n=8$, grupo controle) e diabéticos em uso de insulina $(G 3, n=8)$ da Associação de Diabéticos de Bauru (ADB). Foram submetidos a avaliações clínicas, bioquímicas, pressóricas, antropométricas e a uma sessão de exercício aeróbio ( $60 \%$ a $80 \%$ FC máx $_{\text {) }}$. A glicemia capilar foi mensurada em oito momentos durante a sessão (M1 ao M8). A análise estatística foi descritiva (média \pm desvio padrão), os testes utilizados foram de Kruskal Wallis e Friedman, não paramétricos. Resultados: Participaram 23 indivíduos com idade média 59,35 \pm 14,59 anos, 17 do gênero feminino e seis masculino. As taxas de glicemia do M2 ao M8 foram comparadas ao M1, sendo observadas diminuições significativas nos momentos 4, 5, 6, 7, 8 ( $p \leq 0,05)$. Na análise inicial dos grupos $G 1$ e $G 3$ eram obesos e $G 2$ sobrepeso. Na análise da variação da glicemia durante o exercício foi observado que G1 e G3 diferiram nos momentos 2, 3 e 5, G2 e G3 em todos os momentos ( $p<0,05)$. Houve redução significativa apenas no G2 (grupo controle), nos momentos 4, 5, 6 e 7 ( $p<0,05)$. Conclusão: O exercício tem ação hipoglicemiante, entretanto, nos indivíduos com alteração do metabolismo de carboidratos (G1 e G3), a redução glicêmica não é tão evidente.

Palavras-chave: exercício, diabetes mellitus, glicemia.

\section{ABSTRACT}

Introduction: Physical exercise has been proposed as a non-pharmacological treatment of diabetes due to its hypoglycemic effect. Objective: To investigate the acute effect of exercise on blood glucose in diabetics who use insulin or oral antidiabetic agents. Methods: We have studied patients using oral hypoglycemic agents $(G 1, n=7)$, non-diabetics ( $G 2, n=8$, control group) and patients using insulin $(G 3, n=8)$ at the Diabetic Association of Bauru (ADB). Subjects were submitted to clinical and biochemical evaluation, blood pressure and anthropometric measurements and to a session of aerobic exercise ( $60 \%$ to $80 \% H R_{\text {max }}$. Blood glucose was measured at eight times during the session (M1 to M8). We used descriptive statistical analysis (mean \pm standard deviation), and nonparametric tests used were Kruskal Wallis and Friedman's. Results: Participants were 23 individuals aged $59.35 \pm 14.59$ years, 17 female and six male. The blood glucose levels of $M 2$ to $M 8$ have been compared to M1, with significant reductions in times $4,5,6,7,8(p \leq 0.05)$. In the initial analysis of $G 1$ and $G 3$ were obese and $G 2$ overweight. In the analysis of the variation in blood glucose during the exercise, it was observed that $G 1$ and $G 3$ have differed at times 2, 3 and 5, $G 2$ and $G 3$ at all times ( $p<0.05$ ). There was a significant reduction only in G2 (control group) at times 4, 5, 6 and 7 $(p<0.05)$. Conclusion: Exercise has hypoglycemic action, however, in individuals with altered carbohydrate metabolism (G1 and G3) glycemic reduction is not so evident.

Keywords: exercise, diabetes mellitus, blood glucose.

\section{RESUMEN}

Introducción: Por su efecto hipoglucemiante, el ejercicio físico ha sido propuesto como tratamiento no farmacológico de la diabetes. Objetivo: Investigar el efecto agudo del ejercicio aeróbico en la glucemia capilar de diabéticos que hacían uso de insulina o antidiabéticos orales. Métodos: Hicieron parte del estudio diabéticos que hacían uso de hipoglucemiantes orales ( $G 1, n=7)$, no diabéticos ( $G 2, n=8$, grupo control) y diabéticos que utilizaban insulina $(G 3, n=8)$ de la Asociación de Diabéticos de Bauru (ADB). Los participantes pasaron por evaluaciones clínicas, bioquímicas, presión arterial, antropométricas y por una sesión de ejercicio aeróbico (60\% a 80\% FC máx). Las medidas de glucemia capilar fueron realizadas en ocho momentos (M1-M8) en la sesión. El análisis estadística fue descriptiva (media \pm desviación estándar), con utilización de los testes de Kruskal Wallis y Friedman, no paramétricos. Resultados: Participaron 23 diabéticos (17 mujeres y 6 hombres) con edad media de 59,35 \pm 14,59 años. Los valores de glucemia del M2 al M8 fueron comparados al M1, siendo observadas disminuciones significativas en los momentos $4,5,6,7$ y 8 ( $p \leq 0,05)$. En el análisis inicial, las características de los grupos G1 e G3 fueron de obesidad y, en G2, 
de sobrepeso. En el análisis de la variación de glucemia en el ejercicio, fue observado que el G1 y G3 difirieron en los momentos 2, 3 y 5; el G2 y G3 difirieron en todos los momentos $(p<0,05)$. Además, se constató una reducción significativa solamente en el G2 (grupo control), en los momentos 4, 5, 6 y 7 ( $p<0,05)$. Conclusión: El ejercicio tiene acción hipoglucemiante, sin embargo, en los diabéticos con alteraciones del metabolismo de carbohidratos (G1 y G3), la reducción glucémica es menos evidente.

Palabras clave: ejercicio, diabetes mellitus, glucemia.

\section{INTRODUÇÃO}

O diabetes mellitus (DM) é uma desordem metabólica com alta prevalência na população mundial. Ocorre devido a captação deficiente de glicose pelos tecidos, com isso, o pâncreas produz quantidade insuficiente de insulina, conhecida como diabetes mellitus tipo 1 (DM 1) ou há perda da sensibilidade periférica à insulina, o que incide em $90 \%$ dos casos, levando a altos níveis de glicose circulante, também conhecido como diabetes mellitus tipo 2 (DM 2) $)^{1,2}$.

O DM 1 é classificado como insulino-dependente e aparece como resultado da destruição quase total das células beta do pâncreas levando a deficiência absoluta de insulina. Não há uma causa especifica, mas alguns fatores podem influenciar, como os genéticos, autoimunes e ambientais ${ }^{3}$.

No DM 2 ocorre resistência à insulina levando a diminuição da ação nos tecidos-alvo principalmente no fígado, músculos e tecido adiposo. Tem sua etiologia relacionada com causas herdadas ou adquiridas como obesidade androide, dislipidemia e resistência insulínica, hipertensão arterial (HA) (conhecidas como síndrome metabólica), sedentarismo, fatores genéticos e ambientais ${ }^{2,4,5}$.

Várias pesquisas afirmam que o exercício, juntamente com planejamento alimentar e tratamento farmacológico, tem sido importantes no tratamento do $\mathrm{DM}^{2,4-7}$. O exercício é recomendado para portadores de DM em razão de seus vários efeitos benéficos sobre o risco cardiovascular, controle metabólico, benefícios psicossociais de uma vida menos sedentária, redução da pressão arterial e frequência cardíaca, melhora da captação da glicose, aumento da sensibilidade à ação da insulina ${ }^{2,4-7}$.

Todos os níveis de exercícios físicos podem ser realizados por pacientes com DM tanto aeróbio, resistido e de flexibilidade ${ }^{4,8}$. A frequência, intensidade, duração e o tipo de exercício devem ser prescritos com cautela e precisão. A frequência dos exercícios deve ser maior que três vezes por semana, pois se sabe que o aumento da sensibilidade à insulina, associado ao exercício físico, não permanece por mais de 72 horas $^{9}$.

A progressão do condicionamento físico em diabéticos deve ocorrer levando em consideração idade, capacidade funcional, condição clinica, preferências pessoais e objetivos, ou seja, deve-se respeitar a individualidade biológica do paciente seguindo as recomendações em termos de avaliação, acompanhamento e prescrição de exercícios físicos ${ }^{2,4}$

Recomenda-se ter a preocupação em adequar o exercício e horário de pico da ação da insulina para evitar hipoglicemia, para isso o controle glicêmico deve ser feito durante toda a terapia por meio de automonitoração e ajustes terapêuticos são fundamentais para efeitos benéficos do exercício físico ${ }^{4}$.

Nesse contexto, o objetivo do presente estudo foi comparar o efeito agudo do exercício físico aeróbio sobre a glicemia capilar em dois grupos de indivíduos diabéticos, que fazem uso de insulina ou antidiabéticos orais.

\section{MATERIAIS E MÉTODOS}

Trata-se de um estudo prospectivo, quase experimental. O projeto de pesquisa foi aprovado pelo Comitê de Ética em Pesquisa da Universidade do Sagrado Coração (USC), Bauru, SP, Brasil (protocolo no 164/09) em consoante a declaração de Helsinki.

\section{Casuística}

Participaram 23 indivíduos, com idade entre 21 a 83 anos, do Programa de Extensão Universitário: "Atendimento aos pacientes diabéticos na Associação dos Diabéticos de Bauru", realizado na Associação dos Diabéticos de Bauru (ADB).

Foram incluídos aqueles que apresentaram o laudo do teste ergométrico, indicando negativo para isquemia coronariana e que aceitaram participar do estudo com a assinatura do Termo de Consentimento Livre e Esclarecido.

Os critérios excludentes foram: hipoglicemia (abaixo de 70mg/dl) durante o protocolo de exercício, doença arterial coronariana, insuficiência cardíaca, doença vascular periférica, doenças renais, neurológicas, pulmonares graves incapacitantes e patologias musculoesqueléticas que impedissem a prática do exercício aeróbio.

Segundo os critérios pré-estabelecidos anteriormente, os participantes foram divididos em três grupos sendo grupo 1 (G1) constituído por sete indivíduos diabéticos que utilizavam antidiabéticos orais, grupo 2 (G2) oito indivíduos não diabéticos e grupo 3 (G3) oito indivíduos diabéticos que faziam uso de insulina.

Para realização das medidas da pressão arterial sistólica (PAS) e diastólica (PAD) foram utilizados estetoscópio (Rappaport ${ }^{\circledR}$ - Brasil) e esfigmomanômetro aneroide (Solidor ${ }^{\circledR}$ - China), de acordo com as VI Diretrizes Brasileiras de Hipertensão Arterial ${ }^{10}$. A frequência cardíaca (FC) foi mensurada por meio do frequencímetro Polar ${ }^{\circledR}$ (Finlândia).

Para a avaliação da massa corporal ( $\mathrm{kg}$ ) foi utilizado balança antropométrica (Toledo ${ }^{\circledR}$ - Brasil), com os indivíduos descalços e o mínimo de roupa. A estatura foi medida por meio de estadiômetro. $\mathrm{O}$ índice de massa corpórea (IMC) foi calculado a partir da massa corporal (kg) e estatura $(\mathrm{m})$ pela fórmula IMC = massa corporal $(\mathrm{kg}) /$ estatura $(\mathrm{m})^{2,11}$. Para a circunferência abdominal (CA) foi utilizada fita antropométrica inextensível com precisão de $0,5 \mathrm{~cm}^{12}$.

Foram realizadas análise do colesterol total, triglicerídeos e glicose utilizando o Teste Enzimático Colorimétrico. Os exames laboratoriais foram realizados no Laboratório Tecnolab da cidade de Bauru, SP, Brasil. Para tanto, o sangue dos participantes foi coletado no período matutino, sendo que os mesmos permaneceram em jejum de 12 horas antes da coleta.

Para realização da mensuração da glicemia capilar foi usado o glicosímetro (monitor Accu-Check, Roche ${ }^{\circledR}$ - Alemanha). A glicemia capilar foi avaliada em oito momentos (M): momento um (M1): repouso de 5 min; momento dois (M2): após aquecimento; momento três (M3): 10 min de exercício; momento quatro (M4): 20 min de exercício; momento cinco (M5): 30 min de exercício; momento seis (M6): 40 min de exercício; momento sete (M7): após relaxamento; momento oito (M8): após repouso de 5 min. 


\section{Programa de intervenção}

Os participantes dos três grupos foram submetidos ao protocolo de exercício descrito na figura 1. O protocolo teve a duração de uma hora e meia. A intensidade do exercício foi controlada pela $\mathrm{FC}^{13}$ utilizando um monitor cardíaco (Polar - Finlandia). Antes e após a sessão de exercício foram verificadas a PA e FC.

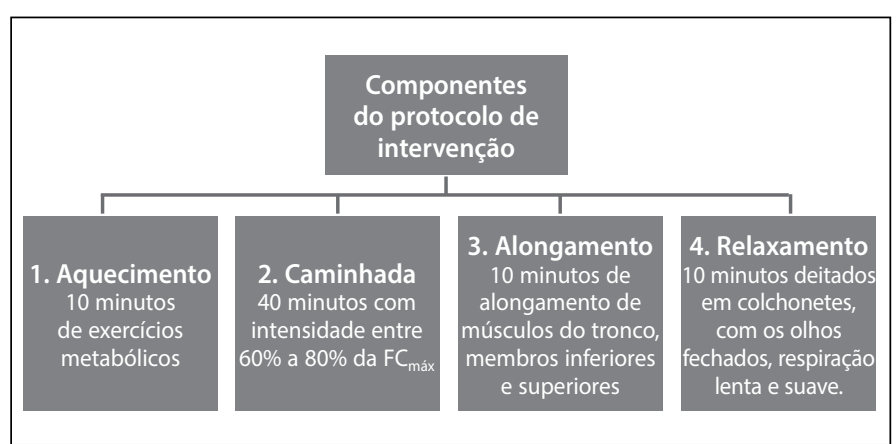

Figura 1. Fluxograma do protocolo de intervenção.

\section{Análise estatística}

Foi realizada análise descritiva, os dados com distribuição normal foram apresentados em média e desvio-padrão, os dados de variáveis com distribuição não paramétrica foram apresentados em mediana e amplitude. Foi utilizado o teste não paramétrico de Kruskal Wallis, para grupos independentes. Utilizou-se o teste não paramétrico de Friedman para amostras pareadas com comparação de n grupos, considerando $p<0,05$.

\section{RESULTADOS}

Foram estudados 23 indivíduos com média de idade 59,35 \pm 14,59 anos, sendo 17 (73\%) do sexo feminino e 6 (26\%) do masculino. A massa corporal dos indivíduos foi em média de 71,30 \pm 15,04 kg, estatura 1,58 \pm 0,08 m, IMC 28,31 \pm 4,76 kg/m² e circunferência abdominal 97,87 \pm 12,74 cm. A média de PAS foi de 126,65 \pm 18,08 mmHg; PAD 73,94 \pm $9,73 \mathrm{mmHg}$ e FC de repouso 76,17 $\pm 12,74 \mathrm{bpm}$. A média do colesterol total foi de 187,89 $\pm 38,78 \mathrm{mg} / \mathrm{dl}$, triglicerídeos 155,46 \pm 106,74 mg/dl e glicose $121,62 \pm 57 \mathrm{ml} / \mathrm{dl}$.

Do grupo total 18 (78,26\%) estavam acima do peso ${ }^{11}$. Em relação a circunferência abdominal $87 \%$ estavam acima dos valores ideais ${ }^{12}$. Observou-se que cinco (21\%) tinham a PAS $\geq 140 \mathrm{mmHg}$ e/ou PAD $\geq 90 \mathrm{mmHg}^{10}$. Foram observados valores acima do ideal de colesterol total (26\%) triglicerídeos e glicose, ambos $43 \%$.

As características iniciais de cada grupo estão mostradas na tabela 1.

Na análise dos grupos observou-se que em G1 seis indivíduos (86\%) e em G3 cinco (62,5\%) estavam acima do peso ideal, em G2 sete (88\%) eram sobrepeso. Em relação à circunferência abdominal, todos os indivíduos do $G 1$ e $G 2$ e cinco $(62,5 \%)$ do $G 3$, estavam acima dos valores ideais ${ }^{14}$.

No G1, a glicose estava acima dos valores de normalidade. No G2, o colesterol total estava acima do esperado. No G3, apenas o colesterol total estava dentro dos valores ideais ${ }^{12}$.

Os valores glicêmicos do protocolo de exercício de todos os indivíduos foram de 170,22 $\pm 80,27 \mathrm{ml} / \mathrm{dl}$ no M1, no M2 foi 156,65 \pm $67,83 \mathrm{ml} / \mathrm{dl} ; 140,61 \pm 67,43 \mathrm{ml} / \mathrm{dl}$ no M3; $128,48 \pm 61,98 \mathrm{ml} / \mathrm{dl}$ no M4; no M5 foi 126,74 $\pm 58,99 \mathrm{ml} / \mathrm{dl}$; no M6 122,39 \pm 56,36ml/dl, no M7 124,65 \pm $51,93 \mathrm{ml} / \mathrm{dl}$ e no $\mathrm{M} 8$ 126,04 $\pm 50,78 \mathrm{ml} / \mathrm{dl}$ (figura 2). Foram comparados todos os momentos em relação ao $\mathrm{M} 1$, sendo observada diminuição significativa nos respectivos momentos $4,5,6,7$ e 8 ( $p \leq 0,05)$.

No G1 as medianas dos valores de glicemia, no M1, foram 129

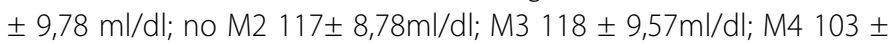
$10,78 \mathrm{ml} / \mathrm{dl} ; \mathrm{M} 5$ 92 \pm 9,21 ml/dl; M6 $101 \pm$ 10,57ml/dl, M7 $104 \pm 11,57 \mathrm{ml} /$ dl e M8 $105 \pm 9,64 \mathrm{ml} / \mathrm{dl}$.
No G2 as medianas dos valores de glicemia, no M1, foram $123 \pm$ 9,78ml/dl; no M2 $118 \pm 8,25 \mathrm{ml} / \mathrm{dl} ; \mathrm{M} 3$ 95,5 \pm 7,12 ml/dl; M4 87,5 \pm $6,31 \mathrm{ml} / \mathrm{dl} ; \mathrm{M} 591 \pm 62,5 \mathrm{ml} / \mathrm{dl} ; \mathrm{M} 689,5 \pm 7,81 \mathrm{ml} / \mathrm{dl}, \mathrm{M} 789 \pm 7,75 \mathrm{ml} / \mathrm{dl}$ e $\mathrm{M} 898,5 \pm 8,5 \mathrm{ml} / \mathrm{dl}$.

No G3 as medianas dos valores de glicemia, no M1, foram 211 \pm 17,68ml/dl; M2 $195 \pm$ 18,56ml/dl; M3 $174 \pm$ 19ml/dl; M4 160,5 \pm 18,75ml/dl; no $164 \pm$ 18,62ml/dl; M6 151,5 \pm 17,43ml/dl, M7 $172 \pm$ $16,62 \mathrm{ml} / \mathrm{dl}$ e $\mathrm{M} 8184,5 \pm 17,56 \mathrm{ml} / \mathrm{dl}$. Houve diferenças entre o G1 e G3 nos momentos 2, 3 e 5 e G2 e G3 em todos os momentos ( $p<0,05)$.

Na análise da variação da glicemia durante o exercício foi observado redução significativa apenas no G2 (grupo controle), nos momentos 4 , 5,6 e $7(p<0,05)$ (figura 3).

Tabela 1. Caracterização dos sujeitos dos grupos 1 (G1) indivíduos diabéticos em uso de antidiabéticos orais, grupo 2 (G2) não diabéticos e grupo 3 (G3) diabéticos em uso de insulina.

\begin{tabular}{|c|c|c|c|c|}
\hline Variáveis & $\mathrm{G1}(\mathrm{n}=7)$ & G2 (n=8) & G3 $(n=8)$ & Valor de $p$ \\
\hline Idade (anos) & $63,29 \pm 7,95$ & $65,38 \pm 9,12$ & $49,88 \pm 19,29$ & $p=0,06$ \\
\hline $\begin{array}{l}\text { Sexo feminino/ } \\
\text { masculino (\%) }\end{array}$ & $71 / 29$ & $100 / 0$ & $62 / 37$ & \\
\hline Massa corporal $(\mathrm{kg})^{*}$ & $80,8 \pm 36,5$ & $63,05 \pm 10,1$ & $77,3 \pm 54,0$ & $p=0,24$ \\
\hline IMC $\left(\mathrm{kg} / \mathrm{m}^{2}\right)^{*}$ & $30,4 \pm 14,5$ & $27,03 \pm 4,0$ & $30,20 \pm 17,42$ & $p=0,19$ \\
\hline$C A(\mathrm{~cm})$ & $102,43 \pm 11,52$ & $96,13 \pm 6,69$ & $95,63 \pm 17,93$ & $p=0,54$ \\
\hline PAS $(\mathrm{mmHg})$ & $125,43 \pm 14,27$ & $124,38 \pm 19,51$ & $130,0 \pm 21,25$ & $p=0,82$ \\
\hline PAD (mmHg) & $73,71 \pm 9,76$ & $71,25 \pm 9,91$ & $76,83 \pm 10,0$ & $p=0,53$ \\
\hline $\mathrm{FC}(\mathrm{bpm})$ & $85,71 \pm 13,73$ & $66,75 \pm 9,32$ & $77,25 \pm 8,26$ & $\begin{array}{c}p=0,006 \\
G 1 \times G 2\end{array}$ \\
\hline $\mathrm{CT}(\mathrm{mg} / \mathrm{dl})$ & $172,6 \pm 29,82$ & $214,53 \pm 36,01$ & $174,63 \pm 37,71$ & $\begin{array}{c}p=0,033 \\
G 2 \times G 3\end{array}$ \\
\hline $\mathrm{TG}(\mathrm{mg} / \mathrm{dl})^{*}$ & $83,0 \pm 139,1$ & $137,0 \pm 75,0$ & $191,0 \pm 426,0$ & $\begin{array}{c}p=0,050 \\
G 1 \times G 3\end{array}$ \\
\hline Glicose $(\mathrm{mg} / \mathrm{dl})^{*}$ & $123,0 \pm 93,5$ & $94,5 \pm 21,6$ & $143,0 \pm 247,0$ & $p=0,33$ \\
\hline
\end{tabular}

*Resultados em mediana e amplitude. IMC = índice de massa corpórea; $C A$ = circunferência abdominal: $P A S=$ pressão arterial sistólica; $\mathrm{PAD}=$ pressão arterial diastólica; $\mathrm{FC}=$ frequência cardíaca; $\mathrm{CT}=$ colesterol total; $\mathrm{TG}=$ triglicerídeo

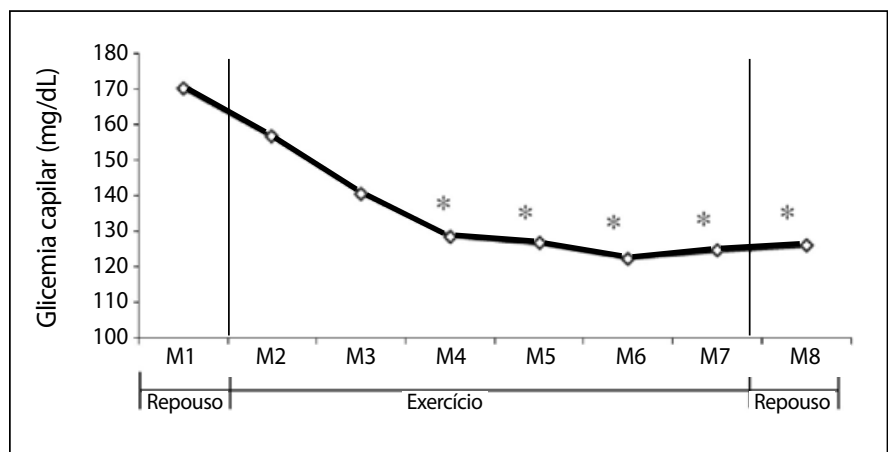

Figura 2. Média da glicemia capilar nos momentos 1 (repouso de 5 min.), 2 (após aquecimento), 3 (10 min. de exercício), 4 (20 min.), 5 (30 min.); 6 (40 min.), 7 (após alongamento e relaxamento) e 8 (após repouso de $5 \mathrm{~min}$.) durante o protocolo de exercício do grupo total $(n=23)$. ${ }^{*} p<0,05$ comparados ao M1.

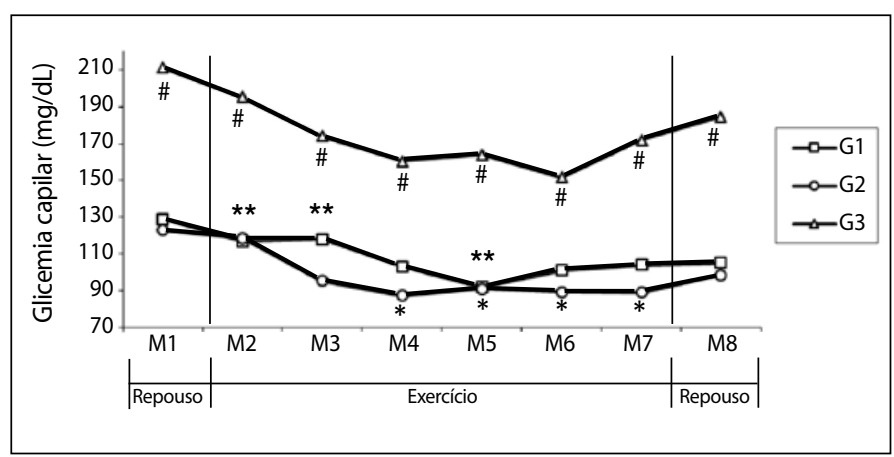

Figura 3. Média da glicemia capilar do grupo 1 (G1 - diabéticos que não usam insulina, $n=7$ ), grupo 2 ( $G 2$ - não diabéticos, $n=8$ ) e grupo 3 (G3 - diabéticos que usam insulina, $n=8$ ) nos momentos 1 (repouso 5 min.), 2 (após aquecimento), 3 (10 min. exercício), 4 (20 min.), 5 (30 min.); 6 (40 min.), 7 (após alongamento e relaxamento) e 8 (após repouso 5 min.) durante o protocolo de exercício. * $\mathrm{p}<0,05$, comparados ao M1 para G2; ** $p<0,05$, comparados G1 e G3; \# $p<0,05$, comparados G2 e G3. 


\section{DISCUSSÃO}

O efeito agudo do exercício foi observado nesse estudo e corrobora com vários autores, independente do tipo de exercício 5-9, 15-17.

A redução da glicemia capilar após uma sessão de exercício físico pode ser explicada devido ao aumento da permeabilidade à glicose nas fibras musculares ativas, mesmo na ausência e/ou deficiência da ação da insulina. Neste sentido, o exercício físico regular aumenta a captação e o metabolismo da glicose pelo músculo, assim como, incrementa a síntese e translocação de Glut-4, transportadores de glicose no tecido adiposo, músculo esquelético e músculo cardíaco ${ }^{19-22}$.

Em estudo realizado por Cambri et al. ${ }^{18}$ foi avaliada a glicemia capilar pré e pós-sessão de exercícios físicos e verificou-se diminuição desta nos indivíduos diabéticos em 78,0\% das sessões analisadas, com variações de 0,4 até 62,5\% em relação à glicemia pré-exercício. Constatou-se ainda, que a redução média da glicemia após as sessões de exercícios físicos foi de 18,0\% ( $p<0,05)$. O presente estudo apresentou dados semelhantes que variaram 18,6\% no G1, 19,9\% no G2 e 12,5\% no G3. Já no estudo de Volpato e Zaboti ${ }^{15}$ houve momentos, quando o exercício foi realizado de maneira contínua, que os níveis de glicose chegaram a diminuir cerca de $50 \%$ do seu valor inicial.

Durante o exercício, o transporte de glicose na célula muscular (GLUT 4) aumenta, assim como a sensibilidade da célula à ação da insulina. Isso ocorre pelo aumento do aporte sanguíneo que é um importante fator regulador permitindo a disponibilidade desse substrato à musculatura. O transporte de glicose ao músculo esquelético ocorre primeiramente por difusão facilitada através dos GLUTs cujos principais mediadores são a insulina e o exercício. Esse aumento na translocação de transportadores de glicose pode ser dependente de insulina ou não. É possível haver translocação de GLUT4 para a membrana muscular durante o exercício mesmo em ausência de insulina ${ }^{5,6,19-22}$

Em indivíduos saudáveis, a ligação da insulina ao seu receptor na membrana plasmática faz com que ocorra a autofosforilação dos resíduos de tirosina do receptor, fosforilação dos substratos do receptor de insulina IRS1 e IRS2 e ativação do fosfatidil inositol 3-quinase. Isso faz com que ocorra a translocação do Glut-4 para o interior da célula ${ }^{5,6}$.

Quando há pratica de exercícios, os mecanismos moleculares envolvidos na translocação dos transportadores de glicose, independentemente da ação da insulina, não são imediatos, porém, não ocorre fosforilação dos receptores nem ativação da PI 3-quinase. Evidências parecem indicar que um mediador do processo de translocação é o cálcio, pois este inicia ou facilita a ativação de moléculas sinalizadoras que leva aos efeitos imediatos e prolongados do exercício sobre o

\section{REFERÊNCIAS}

1. IDF. International Diabetes Federation. The IDF Consensus worldwide definition of the metabolic syndrome. Brussels, Belgium. 2006

2. Vancini RL, Lira CAB. Aspectos gerais do diabetes mellitus e exercício. Centro de Estudos de Fisiologia do Exercício, São Paulo. 2004. 1-15. Disponível em: http://www.isegnet.com.br/siteedit/arquivos/ diabetes\%20Prof.\%20Rodrigo\%20-\%20artigo.pdf. Acesso em 23 de maio de 2013

3. American Diabetes Association. Standards of Medical Care in Diabetes - 2012. Diabetes Care 2012;35 (Suppl 1:) S11-63.

4. De Angelis K, Pureza DY, Flores LJF, Rodrigues B, Melo KFS, Schaan BD, et al. Efeitos fisiológicos do treinamento físico em pacientes portadores de diabetes tipo 1. Arq Bras Endocrinol Metabol. 2006;50(6):1005-13.

5. Barrile SR, Martinelli B, Nicola M, Rino AV, Teixeira MF, Negrato CA. Efeito do exercício físico aeróbio agudo em indivíduos portadores de diabetes mellitus que fazem uso de insulina. Diabetes Clínica 2007;1 1(4):345-51.

6. Irigoyen $M C$, De Angelis $K$, Schann BD, Fiorino P, Michelini LC. Exercício físico no diabetes melito associado à hipertensão arterial sistêmica. Rev Bras Hipertens. 2003 10(2):109-17.

7. Tsalikian E, Mauras N, Beck RW, Tamborlane WV, Janz KF, Chase HP et al. Diabetes Research In Children Network Direcnet Study Group. Impact of Exercise on Overnight Glycemic control in Children with Type 1 Diabetes J Pediatr. 2005;147(4):528-34

8. Ciolac EG, Guimarães GV. Exercício físico e síndrome metabólica. Rev Bras Med Esporte 2004;10(4):319-24.

9. Vancea DMM, Vancea JN, Pires MIF, Reis MA, Moura RB, Dib AS. Efeito da frequência do exercício físico no controle glicêmico e composição corporal de diabéticos tipo 2. Arq Bras Cardiol. 2009;92(1):23-30.

10. Diretrizes Brasileiras de Hipertensão (VI) Sociedade Brasileira de Hipertensão, Sociedade Brasileira de Cardiologia, Sociedade Brasileira de Nefrologia. Arq Bras Cardiol. 2010;95(1supl.1):1-51.

11. Cronk CE, Roche AF. Race and sex-specific reference data for triceps and subscapular skinfolds and weight/stature. Am J Clin Nutr. 1982;35(2):347-54. transporte de glicose no músculo. Outros mediadores podem ser o óxido nítrico, a calicreína e a adenosina ${ }^{5,6,16}$.

A insulina, assim como o exercício, tem efeito hipoglicemiante com melhora na captação de glicose, porém durante o exercício ocorre entrada de glicose na célula independente da ação da insulina ${ }^{5}$. A liberação do cálcio pelo retículo sarcoplasmático durante a contração desencadeia a cascata de sinalização para a translocação dos transportadores de glicose (Glut 4) presentes nas células musculares, ocorrendo assim a entrada de glicose na célula ocasionando o efeito hipoglicemiante ${ }^{6,21}$.

Com indivíduos diabéticos 1 e 2, durante o exercício, a captação de glicose aumenta de forma semelhante à indivíduos saudáveis ${ }^{6}$.

A hipoglicemia induzida pelo exercício pode ocorrer durante, após ou depois de algumas horas. Em indivíduos não diabéticos, durante o exercício, ocorre redução da concentração de insulina no plasma evitando a hipoglicemia. Em indivíduos diabéticos, essa redução não ocorre e, além disso, o exercício aumenta os efeitos da insulina ministrada ocorrendo queda da produção de glicose pelo fígado aumentando consequentemente o uso de glicose pelos músculos em atividade e essa dessincronização leva à hipoglicemia².

Pode-se observar também, tendência ao aumento da glicemia após cessar o exercício. Este estudo utilizou protocolo semelhante ao de um estudo prévio por nosso grupo envolvendo indivíduos diabéticos ${ }^{5,8}$.

Os efeitos do exercício sobre a homeostase glicêmica envolvem mecanismos a curto e longo prazo ${ }^{20}$. Os estudos dos efeitos em longo prazo têm evidenciado benefícios no controle e prevenção do diabetes ${ }^{17,19}$. Os efeitos em curto prazo parecem ser mais evidentes em indivíduos não diabéticos ${ }^{20}$. Fato que corrobora com os resultados encontrados no presente estudo, quando foi realizada a análise entre os grupos, com redução significativa da glicemia durante o exercício apenas do grupo controle.

\section{CONCLUSÃO}

Concluiu-se que o exercício teve ação hipoglicemiante nos indivíduos não diabéticos, entretanto, nos indivíduos com alteração do metabolismo de carboidratos (G1 e G3) a redução glicêmica não foi tão evidente.

\section{AGRADECIMENTOS}

Este estudo foi parcialmente financiado pelo Fundo de Amparo a Pesquisa da Universidade do Sagrado Coração (FAP/USC).

$\overline{\text { Todos os autores declararam não haver qualquer potencial conflito }}$ de interesses referente a este artigo. 